'Instituto de Matemática y Física, Universidad de Talca, Talca, Chile. Programa de Investigación de Excelencia Interdisciplinaria en Envejecimiento Saludable, PIEI-ES 2Departamento de Salud Pública, Facultad de Ciencias de la Salud, Universidad de Talca, Talca, Chile. Programa de Investigación de Excelencia Interdisciplinaria en Envejecimiento Saludable, PIEI-ES aBiometrics PhD.

${ }^{b}$ Cirujano Dentista, Master of Public Health.

'Alumna Interna de Odontología Universidad de Talca.

Fuente de Financiación: sin financiación.

Recibido el 23 de diciembre de 2015, aceptado el 14 de octubre de 2016.

Correspondencia a: Gloria Icaza

Casilla 721 Talca-Chile, Teléfono: 56-712200338 gicaza@utalca.cl

\section{Descripción epidemiológica de la mortalidad por cáncer de mama en mujeres en Chile}

\author{
GLORIA ICAZA ${ }^{1, a}$, LORETO NÚÑEZ $2, b$, HERNA BUGUEÑO ${ }^{2, c}$
}

\section{Epidemiological analysis of breast cancer mortality in women in Chile}

Background: Among women, breast cancer is the leading cause of death due to cancer worldwide. Aim: To describe the epidemiology of breast cancer mortality in Chilean women by age, time trend and explore its ecological association with socio-demographic variables. Material and Methods: Descriptive study of age specific death rates (2009-2013), and time trend analysis of crude mortality rates (1995-2013) using RiskDiff analysis. Additionally, time trend analysis of age specific death rates was done using Jointpoint regression. The relationship between county mortality risk and socio-demographic variables in the period 2001-2008 was done through an ecological analysis. Socio-demographic variables were: education, income, occupation, housing and living in rural areas. Results: Breast cancer mortality in Chilean women increases with age, with a sharp increase from 80 years old on. In the 1995-2013 period the increase in the crude death rate was $21.8 \%$, this increment was due to changes in demographic structure (43.4\%) and decrease in risk (21.7\%). The county relative risk of breast cancer mortality is positively associated with education level and negatively associated with living in rural areas. Conclusions: The risk of dying from breast cancer in women has decreased in the period 1995-2013. Nonetheless, the crude death rate has increased in the same period. At an ecological level (counties), breast cancer mortality in Chile is associated with a higher socioeconomic status, measured by educational level and living in rural areas.

(Rev Med Chile 2017; 145: 106-114)

Key words: Breast Neoplasm; Epidemiology; Mortality; Women; Trends.
E n la mujer, el cáncer de mama es la primera causa de muerte por cáncer a nivel mundial, estimándose 522 mil defunciones en el año 2012, con una tasa estandarizada de mortalidad de 12,9 por 100.000 mujeres y una tasa de incidencia de 43,3 por 100.000 mujeres, lo que corresponde a $25,2 \%$ de la incidencia de cáncer en este grupo ${ }^{1}$. El cáncer de mama en mujeres se ha caracterizado por una alta incidencia en países desarrollados y un aumento de la incidencia en países en desarrollo, como resultado de cambios demográficos y aumento en la prevalencia de factores de riesgo relacionados con estilos de vida y hormonales, tales como aumento de la edad de las mujeres en su primer parto, baja paridad, largo tiempo de exposición al estrógeno, producto de menarquia temprana y menopausia tardía y baja lactancia materna, entre otros ${ }^{2,3}$. Además, en países en desarrollo se observan diferencias entre áreas de residencia urbano y rural ${ }^{4}$.

Al realizar una comparación entre los países pertenecientes a la Organización para la Cooperación y Desarrollo Económico (OCDE), Chile, en etapa de transición demográfica avanzada ${ }^{5}$, se 
encuentra entre los países con tasa baja de mortalidad por cáncer de mama en mujeres, menor que los países miembros de Europa ${ }^{6}$. Con respecto al continente americano, Chile presenta una incidencia y mortalidad por debajo de Estados Unidos de Norteamérica, Canadá, Argentina y Uruguay, y con tasas similares a Colombia y México ${ }^{7}$.

En Chile, el año 2008, el cáncer de mama alcanzó una tasa de mortalidad de 14,5 por 100.000 mujeres, ocupando el segundo lugar de la mortalidad por cáncer ${ }^{8}$. Además, se ha descrito una agregación geográfica de comunas con mayor riesgo de mortalidad por cáncer de mama en mujeres en la Región Metropolitana, la Región de Valparaíso (ambas en la zona central de Chile) y luego en el extremo sur, en la Región de Magallanes; el resto del país presenta riesgo esperado de mortalidad ${ }^{9}$. Estas mismas regiones con exceso de riesgo, destacan con una mayor incidencia estimada, según el primer informe de registros poblacionales de cáncer de Chile del quinquenio 2003-2007 ${ }^{10}$.

El presente estudio utiliza las estadísticas de mortalidad proporcionadas por el Departamento de Estadísticas e Información en Salud (DEIS) del Ministerio de Salud, las cuales han sido consideradas de buena calidad ${ }^{11}$.

El monitoreo de las estadísticas de mortalidad sigue siendo una herramienta útil de vigilancia epidemiológica para medir el progreso de las estrategias preventivas contra el cáncer de mama ${ }^{12}$. Por lo cual, el propósito de este estudio es describir la epidemiología de la mortalidad por cáncer de mama en mujeres de Chile por edad, tendencia temporal y explorar su asociación ecológica con variables sociodemográficas.

\section{Material y Método}

Se realizó un estudio descriptivo de la mortalidad por cáncer de mama (CIE-10, C50) ${ }^{13}$ por edad para el quinquenio 2009-2013, últimos cinco años disponibles a la fecha en el DEIS del Ministerio de Salud ${ }^{14}$, este período de tiempo tiene por objeto circunscribir la descripción por edad a la situación más reciente. Asimismo, se analizó la variación de las tasas crudas de mortalidad entre 1995 y 2013, para lo cual se utilizó la metodología basada en la propuesta de Bashir \& Estève ${ }^{15}$ y disponible en la aplicación web RiskDiff $^{\oplus}$ que desagrega las diferencias observadas de la mortalidad en tres componentes, el riesgo, la estructura y el tamaño de la población.

Este análisis se inicia el año 1995 fecha de creación del Programa Nacional de Cáncer de Mama ${ }^{8}$. Los datos de población se obtuvieron de las proyecciones del Censo $2002^{16} \mathrm{y}$ la actualización de las proyecciones a partir del año $2002^{17}$.

El análisis de la evolución temporal por edad en el período 1995-2013 se realizó con las tasas específicas por decenios de edad, desde los 30 años, mediante una regresión de punto de cambio o jointpoint ${ }^{18,19}$, en el logaritmo de las tasas anuales con errores aleatorios autocorrelacionados, de la cual se obtiene la tasa de cambio porcentual anual (PCA, por su sigla en inglés) y su intervalo de confianza al 95\%, usando el programa Joinpoint Regression Program versión 4.0.4.

Adicionalmente, se exploró la relación del riesgo relativo de mortalidad por cáncer de mama en mujeres con variables sociodemográficas, a través de un análisis ecológico a nivel comunal, utilizando la Razón de Mortalidad Estandarizada suavizada (RMEs) ${ }^{9}$. En Chile, comuna es la unidad administrativa más pequeña de la que se dispone información de mortalidad. Entre las variables sociodemográficas se consideró el porcentaje de ruralidad y variables de educación, ingresos, ocupación y vivienda, elaboradas por el Ministerio de Salud chileno ${ }^{20}$, según metodología utilizada para calcular el Îndice de desarrollo humano (IDH) ${ }^{21}$, basadas en la encuesta de caracterización sociodemográfica (CASEN) del año 2006 22. Para el análisis ecológico se utilizó datos disponibles de la RMEs de cáncer de mama en mujeres a nivel comunal del período 2001-2008 9 .

Finalmente, en el estudio ecológico se llevó a cabo un análisis de correlación lineal simple con el programa $\mathrm{R}$ versión 3.0.2, en 331 de las 345 comunas que cuentan con información sociodemográfica en la encuesta CASEN ${ }^{22}$.

Este estudio utilizó bases de datos secundarios (anónimas) por lo que no fue necesaria su evaluación por un comité de ética científica.

\section{Resultados}

En el año 2013 se registraron 1.389 muertes por cáncer de mama en mujeres, correspondiente a $11,8 \%$ de defunciones oncológicas femeninas. Desde el año 2009 es la primera causa de muerte 
por cáncer en mujeres. La mediana de la edad de muerte para el 2013 fue de 66,0 años.

En el quinquenio 2009-2013 se observa que la mortalidad por cáncer de mama en mujeres aumenta progresivamente con la edad, desde los 25-29 años hasta los 75-79 años, duplicándose en el grupo de 80 años y más. El promedio de muertes por quinquenio de edad es similar entre los 45 y 79 años de edad (promedio 131 casos por quinquenio de edad), en cambio, en el grupo de 80 años y más el promedio de muertes se incrementa 2,6 veces (Figura 1). Adicionalmente, casi un tercio $(32,0 \%)$ de las muertes se produce en mujeres de 75 años y más.

Con respecto a la tendencia de la tasa cruda del año 1995 al 2013, se observa un incremento de $21,8 \%$, esta variación se explica por un descenso de $21,7 \%$ en el riesgo de morir por cáncer de mama en mujeres y un cambio en la estructura demográfica de las mujeres de 43,4\%. El cambio producido en términos de número de casos fue de $49,0 \%$, de los cuales $27,3 \%$ se pueden explicar por cambios en el tamaño poblacional (Figura 2).

$\mathrm{Al}$ analizar la tendencia de las tasas específicas por decenios de edad, se observan tendencias descendentes estadísticamente significativas en la mayoría de los grupos a excepción de 40-49 y 80 y más años. En el grupo 40-49 se observa un cambio en la tendencia en el año 1997, no obstante la tendencia no es significativa en ambos períodos. El decenio de 50-59 años presenta la pendiente más pronunciada con un cambio porcentual anual de $-1,25$ (Tabla 1, Figura 3).

En el análisis ecológico, se observó una correlación negativa significativa entre la RMEs de cáncer de mama en mujeres y el porcentaje de ruralidad, con un coeficiente de correlación $r=-0,51$ (IC 95\% -0,58; -0,42) (Figura 4). Por

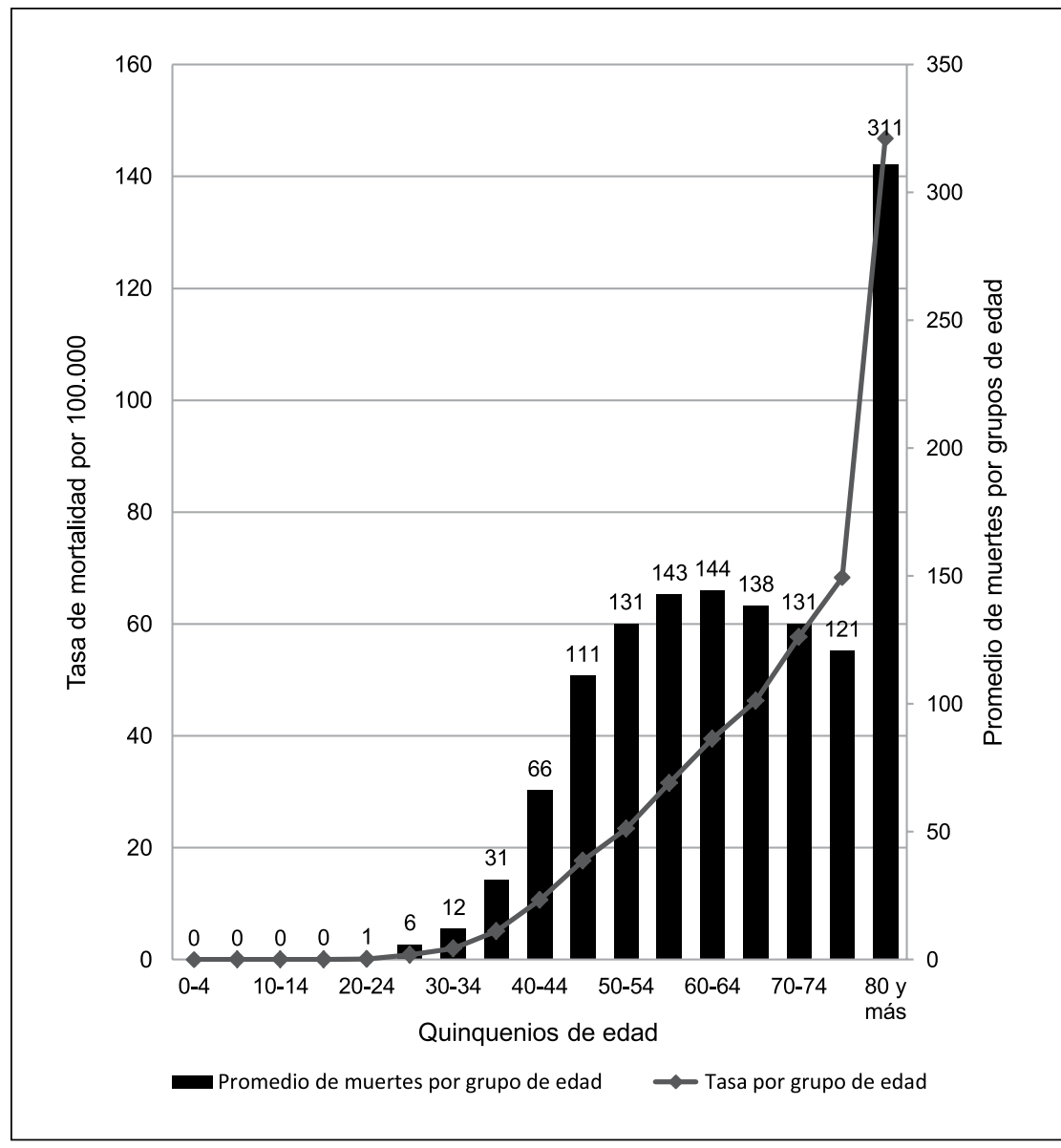

Figura 1. Tasas de mortalidad por cáncer de mama en mujeres por quinquenios de edad, Chile 2009-2013. 
Mortalidad por cáncer de mama - G. Icaza et al

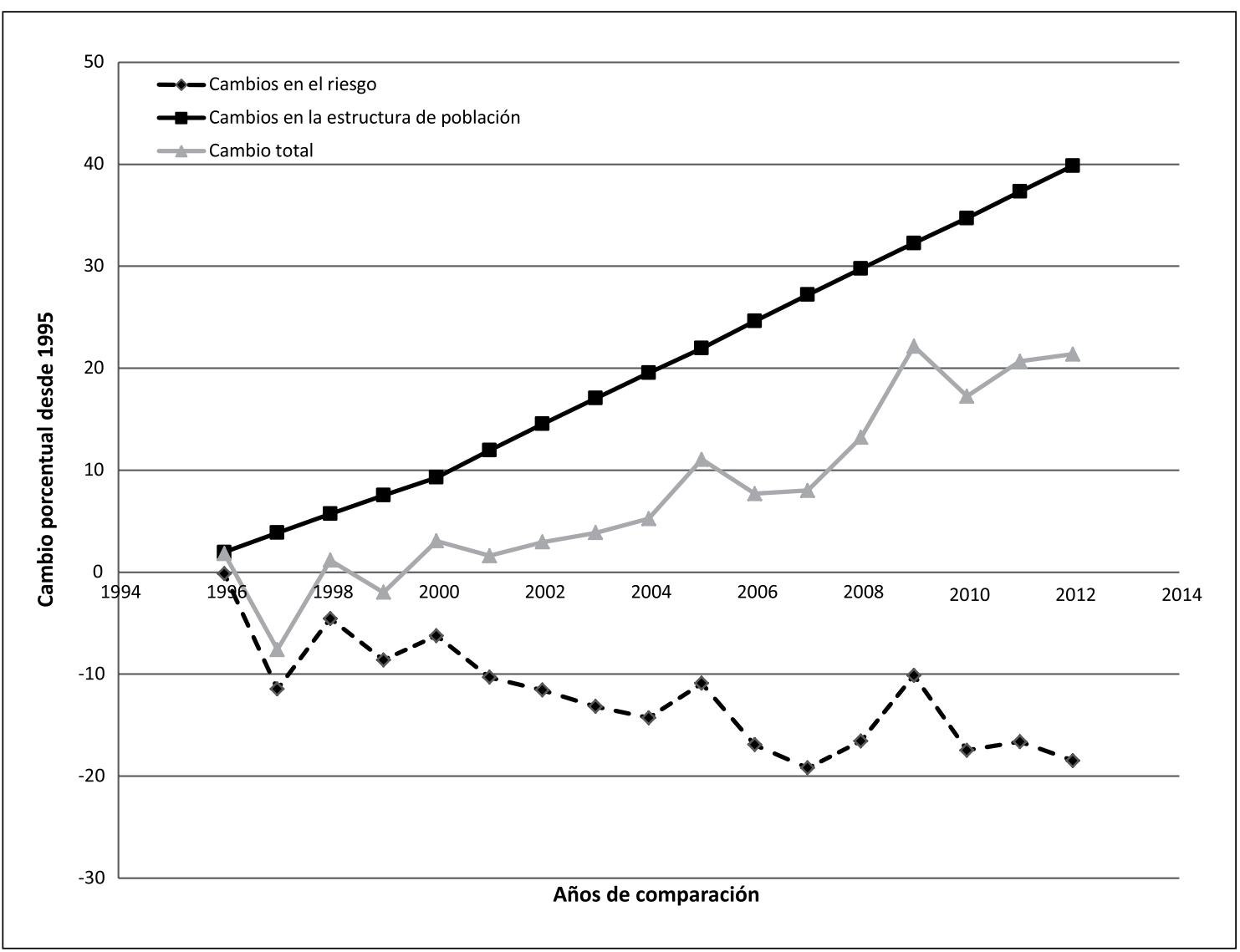

Figura 2. Cambio porcentual en la tasa bruta de cáncer de mama en mujeres en Chile, en comparación con el año de referencia 1995.

Tabla 1. Cambio porcentual anual de las tasas específicas de mortalidad (por 100.000 mujeres) por cáncer de mama por decenios de edad en mujeres

\begin{tabular}{|cccc|}
\hline Decenio de edad & Período & $\begin{array}{c}\text { Cambio porcentual anual } \\
\text { (APC) }\end{array}$ & $\begin{array}{c}\text { Intervalo de confianza } \\
\mathbf{9 5 \%}\end{array}$ \\
\hline $30-39$ & $1995-2013$ & $-0,8^{*}$ & $-1,5 ;-0,1$ \\
\hline $40-49$ & $1995-1997$ & 17,0 & $-36,5 ; 8,5$ \\
\hline $50-59$ & $1997-2013$ & $-0,08$ & $-0,6 ; 0,4$ \\
\hline $60-69$ & $1995-2013$ & $-1,25^{*}$ & $-1,8 ;-0,7$ \\
\hline $70-79$ & $1995-2013$ & $-0,58^{*}$ & $-0,9 ;-0,2$ \\
\hline 80 y más años & $1995-2013$ & $-0,59^{*}$ & $-1,0 ;-0,1$ \\
\hline
\end{tabular}

*Estadísticamente significativo al 5\%. 

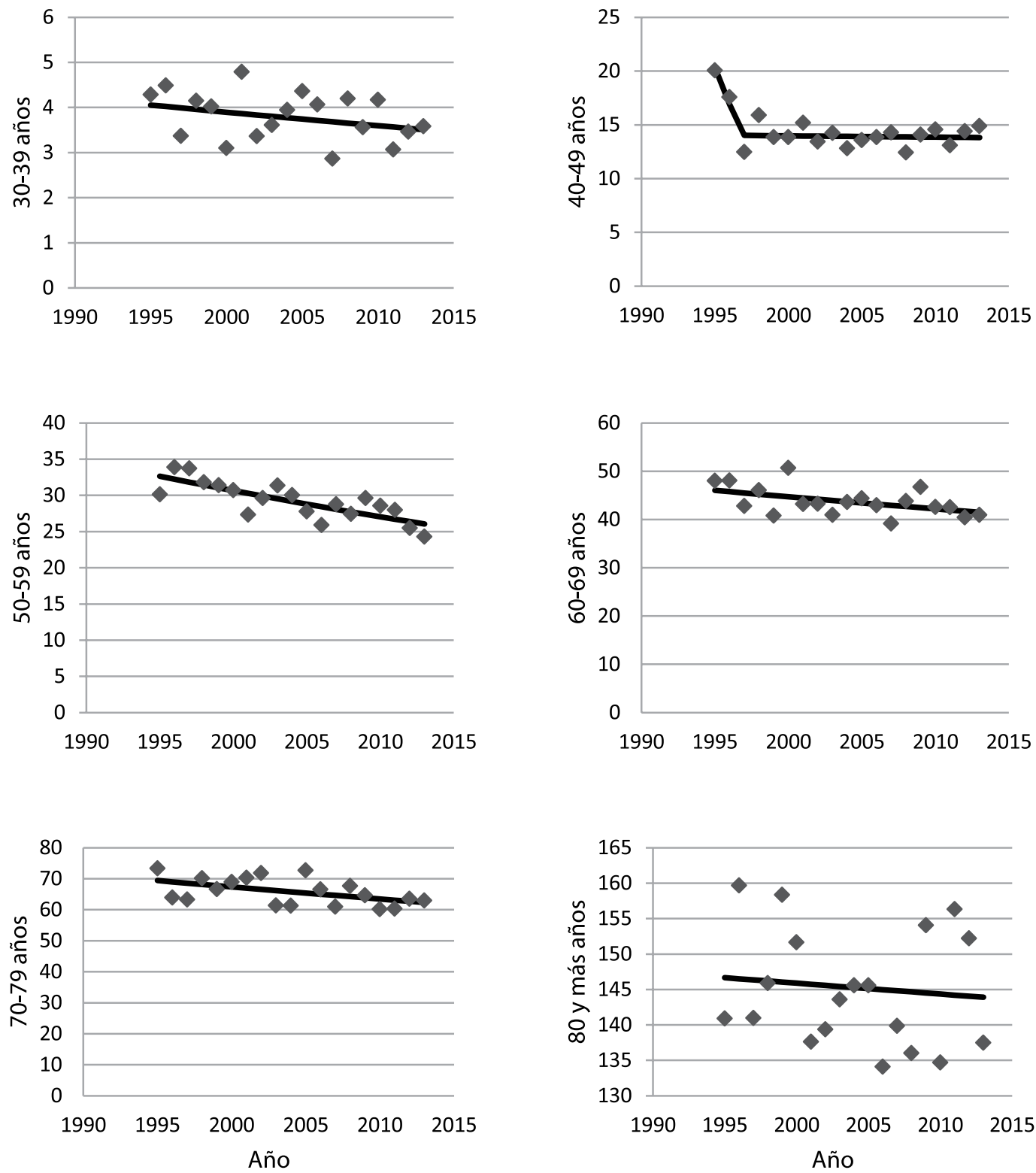

Figura 3. Tasas de mortalidad por 100.000 mujeres por cáncer de mama según decenios de edad en mujeres, Chile 1995-2013.

otro lado, se observa una correlación positiva significativa $r=0,59$ (IC 95\% 0,51; 0,66) entre la RMEs de cáncer de mama y educación, donde 0 significa menor nivel de educación de la comuna (Figura 5). Junto con esto, se observaron relacio- nes similares con ingresos $(\mathrm{r}=0,44$; IC 95\% 0,35; $0,53)$ y vivienda $(r=0,42 ;$ IC $95 \% 0,33 ; 0,51)$. Sin embargo, no se observó una relación estadísticamente significativa con ocupación $(r=0,09$; IC $95 \%-0,02 ; 0,20)$. 


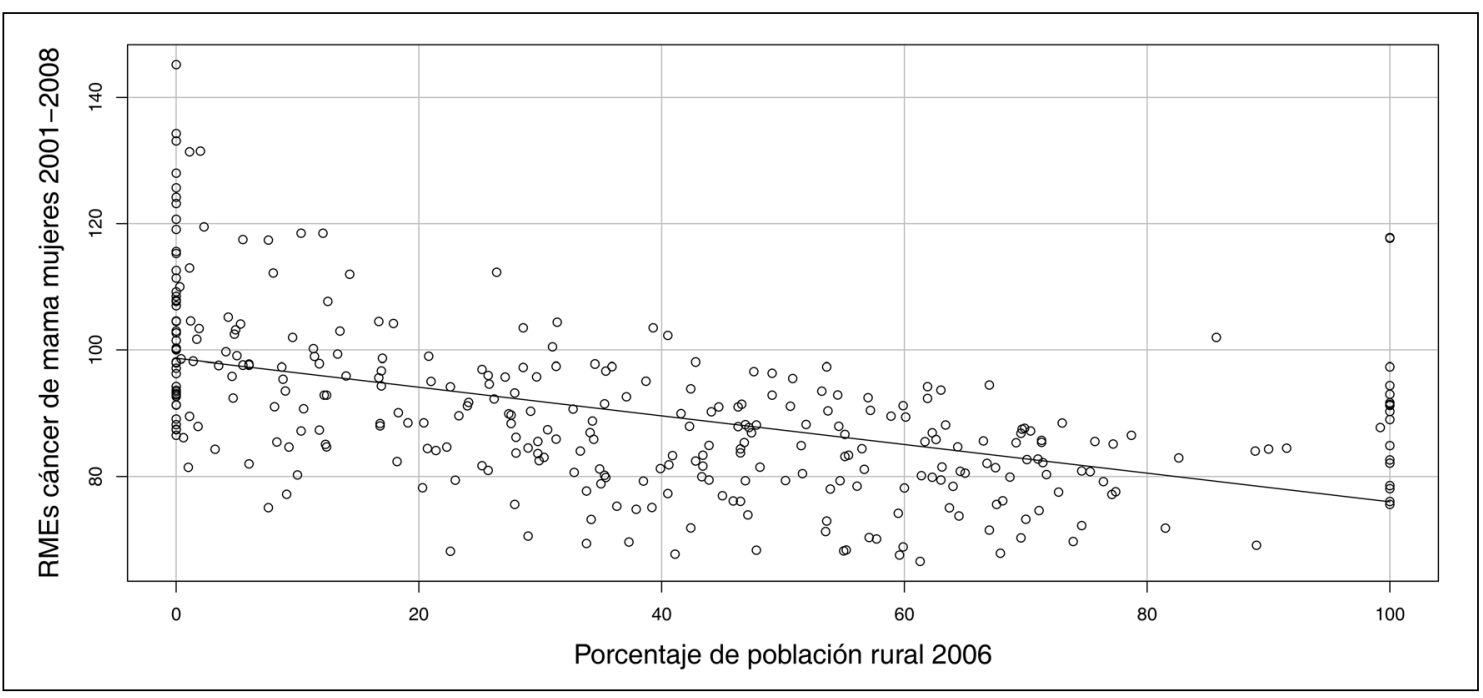

Figura 4. Relación entre RMEs de cáncer de mama en mujeres 2001-2008 y porcentaje de población rural en 2006.

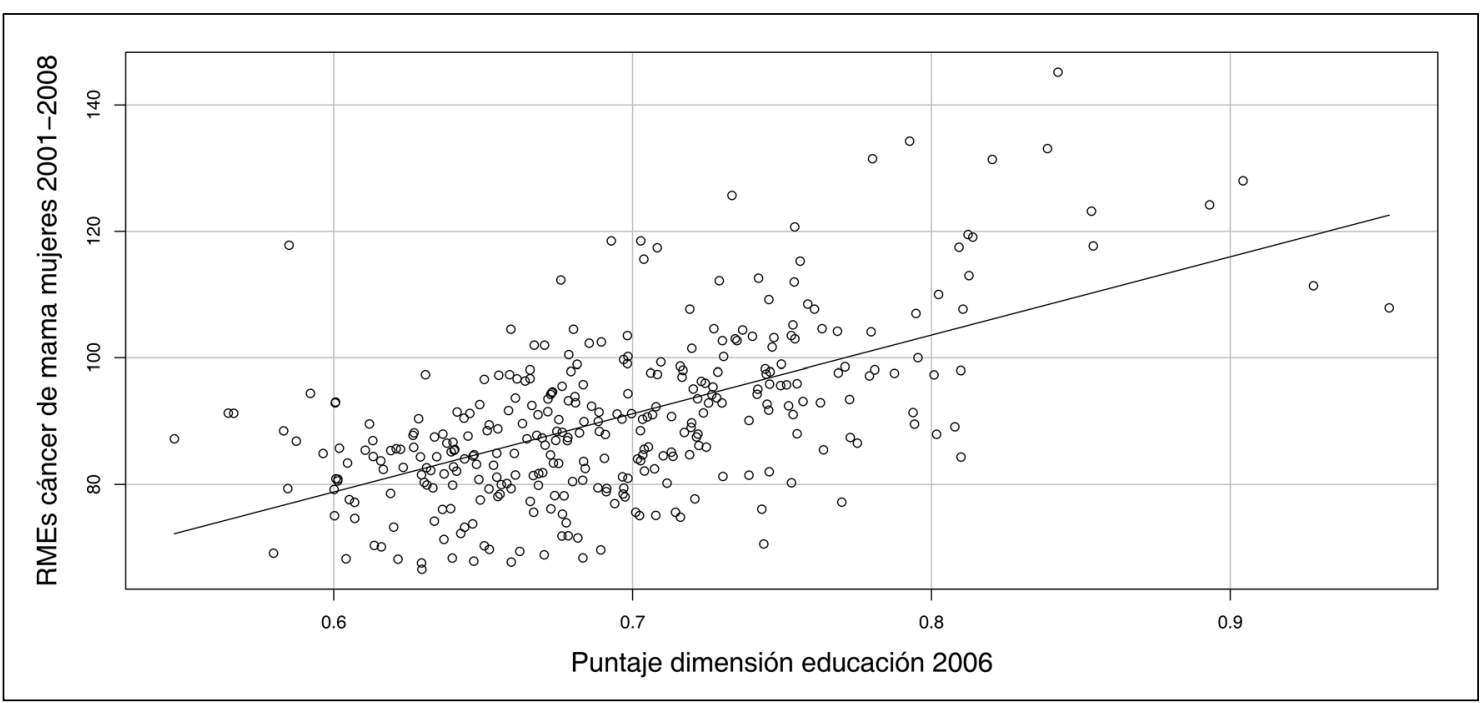

Figura 5. Relación entre RMEs de cáncer de mama en mujeres 2001-2008 y puntaje de dimensión educación en 2006.

\section{Discusión}

La mortalidad por cáncer de mama en mujeres en Chile, en el período 2009-2013, está asociada al envejecimiento, siendo más pronunciada en el grupo de 80 y más años. La tendencia de la tasa bruta de mortalidad por cáncer de mama es ascendente, no obstante el riesgo es descendente. El riesgo relativo de mortalidad por cáncer de mama en mujeres en Chile a nivel comunal, se asoció positivamente con educación y negativamente con el porcentaje de ruralidad.

Dentro de las limitaciones de este trabajo, se puede señalar que si bien Chile es considerado un país con alta calidad de datos de mortalidad ${ }^{11}$, persisten diferencias de calidad entre comunas, área urbano-rural, sexo y edad ${ }^{23}$. Por otra parte, en las asociaciones encontradas a nivel comunal, 
es necesario considerar la falacia ecológica, es decir los resultados no pueden ser extrapolados a nivel individual.

La relación entre mortalidad por cáncer de mama en mujeres y edad ha sido ampliamente reportada, constituyendo ésta su principal factor de riesgo ${ }^{24}$. Por otra parte, el porcentaje de muertes por cáncer de mama en mujeres mayores de 75 años es menor en Chile (32,0\% entre 2009 y 2013) que en Estados Unidos de Norteamérica (37,2\% entre 2007 y 2011$)^{25}$ y Reino Unido (46,0\% entre 2010 y 2012 ${ }^{26}$. Asimismo, la mediana de edad de muerte por cáncer de mama en mujeres en Chile es dos años menor que en Estados Unidos de Norteamérica (66 versus 68 años, respectivamente) ${ }^{25}$. En Chile se reportó una mediana de 65 años para el período 1999-2003, lo cual indicaría que este valor se ha incrementado levemente en los últimos años ${ }^{27}$. Lo anteriormente descrito, muestra que la mortalidad por cáncer de mama en mujeres en Chile se produce a edades inferiores, comparativamente con países desarrollados. Este fenómeno puede deberse a diversas razones que van desde diferencias en prevalencia de factores de riesgo; la estrategia de alto riesgo que ha sido utilizada a nivel nacional, donde se garantiza la mamografía sólo a mujeres de 50 a 59 años $^{28}$, en circunstancia que en Inglaterra la mamografía se ofrece a mujeres de 50 a 70 años y actualmente están estudiando expandirlo a mujeres de 47 a 73 años ${ }^{29}$. También se debe considerar que la cobertura de mamografía es la más baja entre los países de la OCDE 6 . Otras razones para esta mortalidad prematura pueden ser inequidades en el diagnóstico y tratamiento oportuno de la patología ${ }^{27}$.

Este trabajo muestra una disminución en el riesgo de muerte por cáncer de mama en mujeres en Chile, lo cual es reciente, ya que lo publicado señalaba mayormente una tendencia estable ${ }^{30-32}$, salvo publicaciones con datos de la última déca$\mathrm{da}^{9,33}$. Esta tendencia decreciente es producto de la disminución de la mortalidad en los grupos de edad más jóvenes de 30-39 años y en adultas de 50 a 79 años, lo que contribuye a la disminución de la mortalidad prematura por esta causa. La tendencia decreciente puede ser producto de las estrategias implementadas en el país tendientes a mejorar el acceso a servicio, diagnóstico oportuno y tratamiento ${ }^{27,28}$.

En países desarrollados, se ha observado una tendencia decreciente desde los años 90, producto del efecto combinado de prevención primaria, detección temprana y tratamiento ${ }^{3}$. La importancia relativa de esta patología ha aumentado, constituyendo la primera causa de muerte por cáncer en mujeres en Chile desde 2009, con 11,8\% del total de defunciones por cáncer en mujeres el año 2013. Este porcentaje es mayor que lo registrado en Japón $(8,5 \% \text { en } 2006)^{34}$, que destaca con menor mortalidad entre los países de la OCDE el $2009^{6}$ y menor que en Estados Unidos de Norteamérica $(15,3 \% \text { en } 2004)^{34}$. Por lo cual, la carga de enfermedad en Chile estaría aumentando producto, en parte, de los cambios demográficos experimentados en las últimas décadas y la disminución de las tasas de mortalidad en ciertos grupos etarios.

Para continuar con el mejor entendimiento del cáncer de mama en mujeres sería recomendado realizar el análisis de edad, período, cohorte ${ }^{35}$ para monitorear el impacto de políticas públicas.

Con respecto al análisis ecológico, los resultados reflejan que comunas con menor porcentaje de ruralidad y mayor nivel educacional tendrían mayor riesgo de mortalidad por cáncer de mama. En otras palabras, la urbanización y el nivel educacional alto serían factores de riesgo ecológico de mortalidad por cáncer de mama en Chile. Si bien se ha estudiado la etiología del cáncer de mama a nivel individual, aún es necesario abordar cuáles son las causas de la enfermedad a nivel poblacional, que no necesariamente serán las mismas y el análisis ecológico contribuye a dilucidar esta pregunta $^{36}$. Las asociaciones encontradas han sido descritas en la literatura internacional, esto es, la relación del cáncer de mama con el grado de urbanización y con mayor educación ${ }^{4,37}$. Esto podría explicarse por la mayor prevalencia de factores de riesgo de tipo hormonal en mujeres residentes en comunas urbanas y por diferencias en las etapas de la transición epidemiológica a lo largo del país. No obstante, en países desarrollados este mayor riesgo en áreas urbanas se ha trasladado al sector rural, debido a factores relacionados con la detección y acceso a tratamiento oportuno ${ }^{37}$.

Desde el año 1995 que Chile ha estado implementando mejoras sistemáticas en la prevención del cáncer de mama en mujeres ${ }^{27}$, no obstante existen tareas pendientes tales como extender la edad de mamografía y acreditación de los centros de imágenes mamarias ${ }^{8}$, mejorar la cobertura de esta, para alcanzar indicadores similares a los de la $\mathrm{OCDE}^{6}$ o países desarrollados. 
Mantener actualizada la descripción epidemiológica del cáncer de mama en mujeres en Chile es necesaria para la vigilancia epidemiológica que permita tomar decisiones basadas en la mejor evidencia disponible. Se necesita reforzar las políticas públicas tendientes a la prevención del cáncer de mama en mujeres en Chile para que los beneficios de disminución en la tendencia puedan alcanzar a todas las mujeres.

\section{Referencias}

1. Ferlay J, Soerjomataram I, Dikshit R, Eser S, Mathers C, Rebelo $\mathrm{M}$, et al. Cancer incidence and mortality worldwide: sources, methods and major patterns in GLOBOCAN 2012. Int J Cancer 2014. Disponible en: http:// www.ncbi.nlm.nih.gov/pubmed/25220842 [Consultado el 25 de septiembre de 2014].

2. Porter PL. Cáncer de mama en el mundo. Salud Publica Mex 2009; 51(supl 2): 141-6.

3. Bray F, McCarron P, Parkin DM. The changing global patterns of female breast cancer incidence and mortality. Breast Cancer Res 2004; 6: 229-39.

4. Gonzaga CMR, Freitas-Junior R, Souza MR, Curado MP, Freitas NMA. Disparities in female breast cancer mortality rates between urban centers and rural areas of Brazil: ecological time-series study. Breast 2014; 23 : 180-7.

5. Centro Latinoamericano y Caribeño de Demografía. Cambios en la estructura poblacional: Una pirámide que exige nuevas miradas. Santiago; División de Población de CEPAL; 2005. Disponible en: http://www.cepal.org/ celade/noticias/noticias/2/23462/PyDTD_1.pdf [Consultado el 26 de febrero de 2015].

6. Organización para la Cooperación y el Desarrollo Económicos. Health at a Glance 2013: OECD Indicators. Paris: OECD Publishing; 2013. Disponible en: http:// www.oecd.org/health/health-systems/health-at-a-glance. htm [Consultado el 24 de julio de 2014].

7. Ferlay J, Soerjomataram I, Dikshit R, Eser S, Mathers C, Rebelo M, et al. GLOBOCAN 2012 v1.0, Cancer incidence and mortality worldwide: IARC CancerBase No. 11. Lyon, France: International Agency for Research on Cancer 2014. Disponible en: http://globocan.iarc.fr [Consultado el 24 de julio de 2014].

8. Prieto M. Epidemiología del cáncer de mama en Chile. Rev Med Clin Condes 2011; 22 (4): 428-35.

9. Icaza G, Núñez L, Torres-Avilés F, Díaz N, Villarroel JE, Soto A, et al. Atlas de mortalidad en Chile, 2001- 2008. Talca Chile: Editorial Universidad de Talca; 2013. p. 15-65.
10. Ministerio de Salud. Primer informe de registros poblacionales de cáncer de Chile. Quinquenio 2003-2007. Santiago de Chile: Unidad de Estudios y Vigilancia de Enfermedades No Transmisibles; 2012. Disponible en: http://epi.minsal.cl/epi/0notransmisibles/cancer/ INFORME\%20RPC\%20CHILE\%202003-2007,\%20 UNIDAD\%20VENT,\%20DEPTO.EPIDEMIOLOGIA-MINSAL,13.04.2012.pdf [Consultado el 16 de diciembre de 2014].

11. Mahapatra P, Shibuya K, López AD, Coullare F, Norzon FC, Rao C, et al. Civil registration systems and vital statistics: successes and missed opportunities. Lancet 2007; 370: 1653-63.

12. Jatoi I, Miller AB. Why is breast-cancer mortality declining? Lancet Oncol 2003; 4: 251-4.

13. Organización Mundial de la Salud. Clasificación estadística internacional de enfermedades y problemas relacionados con la salud. $10^{\mathrm{a}}$ revisión. v.3. Lista tabular. Washington, D.C.: OPS. (Publicación científica 554), 1995.

14. Departamento de Estadísticas e Información en Salud. Bases de datos Defunciones. Santiago de Chile: Ministerio de Salud; 1995-2013. Disponoble en: http://www. deis.cl/ [Consultado el 6 de julio de 2015].

15. Bashir SA, Estève, J. Analysing the difference due to risk and demographic factors for incidence or mortality. International Journal of Epidemiology 2000; 29: 878-84.

16. Instituto Nacional de Estadísticas-Centro Latinoamericano y Caribeño de Demografía. Chile: Proyecciones y estimaciones de población. Total país 1950-2050, 2004. Disponible en http://www.deis.cl [Consultado el 12 de marzo de 2010].

17. Departamento de Estadísticas e Información en Salud. Análisis de situación nuevas proyecciones de población INE, comparación con las proyecciones basadas en el Censo 2002 y recomendaciones para su utilización. Actualización mayo de 2015.

18. Kim H, Fay M. Permutation tests for joinpoint regression with applications to cancer rates. Stat Med 2000; 351: 335-51.

19. Vives A, Valdivia G, Marshall G. Cambios recientes en la mortalidad por cáncer de próstata en Chile: estudio de tendencias en el período 1995-2001. Rev Med Chile 2004; 132: 579-87.

20. Diagnósticos regionales de salud con enfoque DSS. Santiago de Chile: Departamento de Epidemiología, Ministerio de Salud; 2009. Disponible en: http://epi. minsal.cl/estudios-y-encuestas-poblacionales/diagnosticos-regionales/ [Consultado el 16 de diciembre de 2014]. 
21. Programa de Naciones Unidas para el Desarrollo. Índice de desarrollo humano en Chile 1990-1998. Santiago de Chile: Naciones Unidas 1999. Disponible en: http:// www.desarrollohumano.cl/otraspub/Pub03/todo.pdf [Consultado el 16 de diciembre de 2014].

22. Encuesta CASEN. Santiago de Chile: Ministerio de Desarrollo Social; 2006. Disponible en: http://observatorio. ministeriodesarrollosocial.gob.cl/casen/casen_obj.php [Consultado el 16 de diciembre de 2014].

23. Núñez L, Icaza G. Calidad de las estadísticas de mortalidad en Chile, 1997-2003. Rev Med Chile 2006; 134: 1191-6.

24. Kamangar F, Dores G, William A. Patterns of Cancer Incidence, Mortality, and Prevalence Across Five Continents: Defining Priorities to Reduce Cancer Disparities in different Geographic Regions of the World. J Clin Oncol 2006; 24: 2137-50.

25. SEER. Stat fact sheets: Breast Cancer. Bethesda (MD): National Cancer Institute; 1973-2011. Disponible en: http://seer.cancer.gov/statfacts/html/breast.html [Consultado el 6 de noviembre de 2014].

26. Cancer Research UK. Breast cancer mortality statistics. London; 2014. Disponible en: http://www.cancerresearchuk.org/cancer-info/cancerstats/types/breast/ mortality/\#age [Consultado el 23 de octubre de 2014].

27. Prieto M. Situación epidemiológica del cáncer de mama en Chile 1994-2003. Rev Med Clin Condes 2006; 17 (4): 142-8.

28. Ministerio de Salud. Guía Clínica AUGE. Examen Medicina Preventiva. Santiago de Chile: División de Prevención y Control de Enfermedades; 2013. Disponible en: http://web.minsal.cl/sites/default/files/files/ GPC\%20Medicina\%20Preventiva.pdf [Consultado el 23 de marzo de 2015].

29. Cancer Research UK. Breast cancer screening in the UK.
London; 2014. Disponible en: http://www.cancerresearchuk.org/about-cancer/type/breast-cancer/about/ screening/who-is-screened-for-breast-cancer\#benefits [Consultado el 8 de agosto de 2016].

30. Lozano-Ascencio R, Gómez-Dantés H, Lewis S, Torres-Sánchez L, López-Carrillo L. Tendencias del cáncer de mama en América Latina y El Caribe. Salud Publica Mex 2009; 51: 147-56.

31. Bosetti C, Malvezzi M, Chatenoud L, Negri E, Levi F, La Vecchia C. Trends in cancer mortality in the Americas, 1970-2000. Ann Oncol 2005; 16: 489-511.

32. Ministerio de Salud. Objetivos Sanitarios para Chile 2000-2010. Santiago de Chile: Departamento de Epidemiología; 2002. Disponible en: http://epi.minsal.cl/epi/ html/sdesalud/OS/OS2000_2010.htm [Consultado el 17 de octubre de 2013].

33. Luciani S, Cabanes A, Prieto-Lara E, Gawryszewski V. Cervical and female breast cancers in the Americas: current situation and opportunities for action. Bull World Health Organ 2013; 91: 640-9.

34. Saika K, Sobue T. Epidemiology of Breast Cancer in Japan and the US. JMAJ 2009; 52: 39-44.

35. Rosenberg PS, Anderson WF. Age-period-cohort models in cancer surveillance research: ready for prime time? Cancer Epidemiol Biomarkers Prev 2011; 20 (7): 1263-8.

36. Rose G. Sick individuals and sick populations. Int J Epidemiol 1985; 14: 32-8.

37. Singh GK, Williams SD, Siahpush M, Mulhollen A. Socioeconomic, Rural-Urban, and Racial Inequalities in US Cancer Mortality: Part I-All Cancers and Lung Cancer and Part II-Colorectal, Prostate, Breast, and Cervical Cancers. J Cancer Epidemiol. 2011 Disponible en: http://www.pubmedcentral.nih.gov/articlerender. fcgi artid $=3307012 \&$ tool $=$ pmcentrez\&rendertype $=$ abs tract [Consultado el 12 de diciembre de 2014]. 